\title{
Cálculo da força de Casimir
}

\author{
Rouver, A. N.; Orlando, M. T. D. ${ }^{1}$ \\ 1 Programa de Pós-Graduação em Engenharia Mecânica, Universidade Federal do Espírito Santo, Vitória, ES, Brasil. \\ *e-mail: arouver@gmail.com
}

\begin{abstract}
Resumo
Através da quantização do campo eletromagnético, nota-se que a energia no campo eletromagnético, quando existem zero fótons presentes, apresenta flutuações quânticas, fazendo com que a energia do vácuo, também chamada de energia de ponto zero, seja não nula. Em 1948, Casimir previu a existência de uma força atrativa entre duas placas condutoras, paralelas e eletricamente neutras e que esta força pode ser interpretada como flutuações da energia de ponto zero das ondas eletromagnéticas. Esse efeito ficou conhecido como Efeito Casimir. Este trabalho busca descrever o passo a passo para o cálculo da força de Casimir, discutindo as considerações feitas e as ferramentas matemáticas utilizadas.
\end{abstract}

\begin{abstract}
By quantization of the electromagnetic field, note that the energy in the electromagnetic field, when there are zero photons present, has quantum fluctuations, causing the vacuum energy, also called zero point energy, is not zero. In 1948, Casimir predicted the existence of an attractive force between two conductive parallel and electrically neutral plates and that this force can be interpreted as energy fluctuations of zero point of electromagnetic waves. This effect became known as the Casimir effect. This paper seeks to describe the step by step to calculate the Casimir force, discussing the considerations and the mathematical tools used.
\end{abstract}

Keywords (Palavras chaves): Casimir effect, vacuum energy, electromagnetic field.

\section{Introdução}

Através da quantização do campo eletromagnético, nota -se que o hamiltoniano do campo eletromagnético quantizado representa uma soma de hamiltonianos formalmente análogos ao do oscilador harmônico.

$$
H=\frac{1}{2} \int d k^{3} \sum_{\lambda=1}^{2}\left(2 \hat{a}_{k \lambda}^{\dagger} \hat{a}_{k \lambda}+1\right) \hbar \omega_{k}
$$

Esta equação representa a soma do número de fótons em cada modo multiplicado pela energia de um fóton em cada um dos modos. Além disso,

$$
E_{0}=\frac{1}{2} \sum_{\lambda=1}^{2} \hbar \omega_{k}
$$

onde $\lambda=1,2$ representa as duas polarizações possivels. Vale ressaltar que o calibre de Coulomb $(\vec{\nabla} \cdot \vec{A}=0)$ imprime uma condição de transversalidade para o campo, onde os vetores de polarização são perpendiculares ao vetor de onda, descartando a possibilidade da presença de fótons longitudinais.
A Equação (2) é a energia das flutuações de vácuo em cada um dos modos. Então, pode-se dizer que o hamiltoniano para o campo eletromagnético quantizado consiste no somatório de infinitos termos de hamiltoniano de osciladores harmônicos descrevendo o comportamento de uma oscilação em cada ponto do espaço.

A Equação (2) é a energia no campo eletromagnético, quando existem zero fótons presentes, também chamada de energia do vácuo. Uma manifestação macroscópica da energia do vácuo é conhecida como efeito Casimir.

Em 1948, Casimir e Polder [1] mostraram que, em consequência da velocidade finita de propagação do campo eletromagnético, a energia de interação Interatômica diminuía com o inverso da sexta potência da distância entre os átomos quando estes estavam bem próximos e com o inverso da sétima potência quando estes estavam a grandes distâncias. Em 1992, numa carta a Milonni [2], Casimir relata o encontro que teve com Niels Bohr a cerca de sua recente descoberta. Nesse encontro, Ihe foi sugerido por Bohr considerar a energia de ponto zero do vácuo: 
"No verão ou outono de 1947 (mas não estou absolutamente certo de que não tenha sido um pouco antes ou depois), mencionei meus resultados a Niels Bohr durante uma caminhada. 'Isto é ótimo' disse ele. 'Isto é algo novo'. Disse-lhe que estava intrigado com a forma extremamente simples das expressões para a interação a grandes distâncias e ele resmungou algo sobre a energia do ponto zero. Isto foi tudo, mas colocou-me em uma nova pista.

Descobri que calcular as variações da energia do ponto zero leva realmente aos mesmos resultados dos cálculos que fiz com Polder...

Em 29 de maio de 1948 apresentei meu trabalho sobre a atração entre duas placas perfeitamente condutoras à Academia Real Holandesa de Artes e Ciências. O trabalho foi publicado no decorrer daquele ano."

Então, também em 1948, Casimir [3] previu a existência de uma força atrativa entre duas placas condutoras, paralelas e eletricamente neutras e que esta força pode ser interpretada como flutuações da energia de ponto zero das ondas eletromagnéticas. Esse efeito ficou conhecido como Efeito Casimir.

\section{CÁLCULO DA FORÇA DE CASIMIR}

O potencial vetor $\vec{A}$ associado a uma onda eletromagnética no vácuo pode ser descrito pela equação de onda

$$
\frac{1}{c^{2}} \frac{\partial^{2} \vec{A}}{\partial t^{2}}-\nabla^{2} \vec{A}=0
$$

Usando o método de separação de variáveis, se faz

$$
\vec{A}(\vec{r}, t)=\vec{v}(\vec{r}) q(t)
$$

onde $q(t)$ é uma função que depende somente do tempo e $\vec{v}(\vec{r})$ é uma função que depende somente de $\vec{r}$. Substituindo a Equação (4) na Equação (3),

$$
\frac{1}{c^{2}} \frac{1}{q(t)} \frac{\partial^{2} q(t)}{\partial t^{2}}=\frac{1}{\vec{v}(\vec{r})} \nabla^{2} \vec{v}(\vec{r})
$$

Observe que o lado direito depende apenas de $\vec{r}$, enquanto o lado esquerdo depende apenas do tempo. Portanto os dois lados devem ser igualados a uma constante $-k^{2}$. Dessa forma, o lado direito da Equação (5) se torna

$$
\frac{1}{\vec{v}(\vec{r})} \nabla^{2} \vec{v}(\vec{r})=-k^{2}
$$

O sinal de menos deve-se ao fato dos autovalores do Laplaciano serem negativos. Já o lado direito da Equação (3.3) se torna

$$
\frac{1}{c^{2}} \frac{1}{q(t)} \frac{\partial^{2} q(t)}{\partial t^{2}}=-k^{2}
$$

Fazendo $\omega=c k$ (conhecida como relação de dispersão), segue que

$$
\frac{\partial^{2} q(t)}{\partial t^{2}}+\omega^{2} q(t)=0
$$

Agora, considera-se uma cavidade retangular de lados $L_{x}, L_{y}$ e $L_{z}$ (Figura 1),

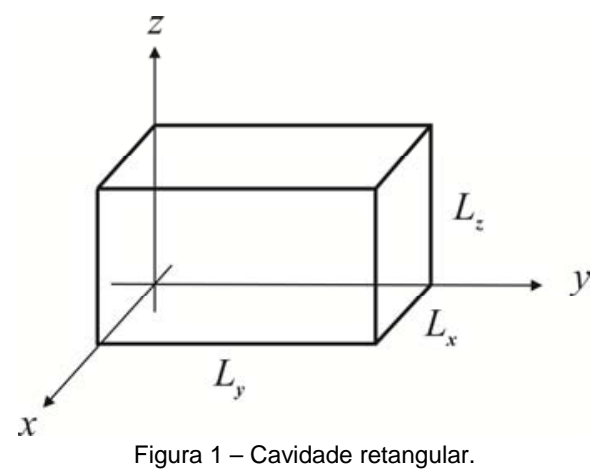

onde $\vec{v}$ pode ser escrito em função das suas coordenadas, ou seja,

$$
\vec{v}(\vec{r})=X(\vec{x}) Y(\vec{y}) Z(\vec{z})
$$

Substituindo a Equação (9) na Equação (6),

$$
\frac{1}{X} \frac{\partial^{2} X}{\partial x^{2}}+\frac{1}{Y} \frac{\partial^{2} X}{\partial x^{2}}+\frac{1}{Z} \frac{\partial^{2} X}{\partial x^{2}}=-k^{2}
$$

Como cada termo depende de uma variável diferente, podem ser igualados a constantes de forma que

$$
\frac{1}{X} \frac{\partial^{2} X}{\partial x^{2}}=-k_{x}^{2} \quad \frac{1}{Y} \frac{\partial^{2} X}{\partial x^{2}}=-k_{y}^{2} \quad \frac{1}{Z} \frac{\partial^{2} X}{\partial x^{2}}=-k_{z}^{2}
$$

que tem como soluções, respectivamente

$$
\begin{gathered}
X=A \sin \left(k_{x} x\right)+B \cos \left(k_{x} x\right) \\
Y=C \sin \left(k_{y} y\right)+D \cos \left(k_{y} y\right) \\
Z=E \sin \left(k_{z} z\right)+F \cos \left(k_{z} z\right)
\end{gathered}
$$

Note que

$$
k=\sqrt{k_{x}^{2}+k_{y}^{2}+k_{z}^{2}}
$$

Reescreve-se $\vec{v}(\vec{r})$ como

$$
v_{i}=\left(A_{i} \sin \left(k_{x} x\right)+B_{i} \cos \left(k_{x} x\right)\right)\left(C_{i} \sin \left(k_{y} y\right)+D_{i} \cos \left(k_{y} y\right)\right)\left(E_{i} \sin \left(k_{z} z\right)+F_{i} \cos \left(k_{z} z\right)\right)
$$




\section{Encontro Científico de Física Aplicada}

Como o potencial deve se anular no interior da cavidade, as condições de contorno serão dadas por

$$
\operatorname{Em} x=0 \text { e } x=L_{x}\left\{\begin{array}{l}
v_{y}=0 \\
v_{z}=0
\end{array} \quad \operatorname{Em} x=0 \text { e } x=L_{x}\left\{\begin{array}{l}
v_{y}=0 \\
v_{z}=0
\end{array} \quad \text { Em } x=0 \text { e } x=L_{x}\left\{\begin{array}{l}
v_{y}=0 \\
v_{z}=0
\end{array}\right.\right.\right.
$$

Portanto, em $y=0$ a Equação (15), com $i=x$, se torna

$$
0=\left(A_{x} \sin \left(k_{x} x\right)+B_{x} \cos \left(k_{x} x\right)\right) D_{x}\left(E_{x} \sin \left(k_{z} z\right)+F_{x} \cos \left(k_{z} z\right)\right)
$$

Isto implica em $D=0$. Agora, em $z=0$,

$$
0=\left(A_{x} \sin \left(k_{x} x\right)+B_{x} \cos \left(k_{x} x\right)\right) C_{x} \sin \left(k_{y} y\right) F_{x}
$$

Isto implica em $F x=0$. Dessa forma, agora a Equação (15) fica

$$
v_{x}=\left(A_{x} \sin \left(k_{x} x\right)+B_{x} \cos \left(k_{x} x\right)\right) C_{x} \sin \left(k_{y} y\right) E_{x} \sin \left(k_{z} z\right)
$$

Analogamente, é possível obter

$$
\begin{gathered}
v_{y}=A_{y} \sin \left(k_{x} x\right)\left(C_{y} \sin \left(k_{y} y\right)+D_{y} \cos \left(k_{y} y\right)\right) E_{y} \sin \left(k_{z} z\right) \\
v_{z}=A_{z} \sin \left(k_{x} x\right) C_{x} \sin \left(k_{y} y\right)\left(E_{z} \sin \left(k_{z} z\right)+F_{z} \cos \left(k_{z} z\right)\right)
\end{gathered}
$$

Usando o calibre de Coulomb, $\vec{\nabla} \cdot \vec{A}=0$. O que implica em

$$
\frac{\partial v_{x}}{\partial x}+\frac{\partial v_{y}}{\partial y}+\frac{\partial v_{z}}{\partial z}=0
$$

Daí,

$$
\begin{aligned}
k_{x}\left(A_{x} \cos \left(k_{x} x\right)\right. & \left.-B_{x} \sin \left(k_{x} x\right)\right) C_{x} \sin \left(k_{y} y\right) E_{x} \sin \left(k_{z} z\right)+A_{y} \sin \left(k_{x} x\right) k_{y}\left(C_{y} \cos \left(k_{y} y\right)-D_{y} \sin \left(k_{y} y\right)\right) E_{y} \sin \left(k_{z} z\right) \\
& +A_{z} \sin \left(k_{x} x\right) C_{z} \sin \left(k_{y} y\right) k_{z}\left(E_{z} \cos \left(k_{z} z\right)-F_{z} \sin \left(k_{z} z\right)\right)=0
\end{aligned}
$$

Esta equação deve ser satisfeita em qualquer ponto da cavidade, inclusive em $(x=0, y, z),(x, y=0, z)$ e $(x, y, z=0)$. Para $(x=0, y, z)$,

$$
k_{x} A_{x} C_{x} \sin \left(k_{y} y\right) \sin \left(k_{z} z\right)=0
$$

Para $(x, y=0, z)$ e $(x, y, z=0)$, respectivamente,

$$
\begin{aligned}
& A_{y} \sin \left(k_{x} x\right) k_{y} C_{y} E_{y} \sin \left(k_{z} z\right)=0 \\
& A_{z} \sin \left(k_{x} x\right) C_{z} \sin \left(k_{y} y\right) k_{z} E_{z}=0
\end{aligned}
$$

Portanto é necessário ter $A_{i} C_{i} E_{i}=0$. Logo, a Equação (22) é escrita como

$$
\begin{gathered}
k_{x} B_{x} \sin \left(k_{x} x\right) C_{x} \sin \left(k_{y} y\right) E_{x} \sin \left(k_{z} z\right)+A_{y} \sin \left(k_{x} x\right) k_{y} D_{y} \sin \left(k_{y} y\right) E_{y} \sin \left(k_{z} z\right) \\
+A_{z} \sin \left(k_{x} x\right) C_{z} \sin \left(k_{y} y\right) k_{z} F_{z} \sin \left(k_{z} z\right)=0
\end{gathered}
$$

e as Equações (18-20) se tornam

$$
\begin{gathered}
v_{x}=B_{x} \cos \left(k_{x} x\right) C_{x} \sin \left(k_{y} y\right) E_{x} \sin \left(k_{z} z\right) \\
v_{y}=A_{y} \sin \left(k_{x} x\right) D_{y} \cos \left(k_{y} y\right) E_{y} \sin \left(k_{z} z\right) \\
v_{z}=A_{z} \sin \left(k_{x} x\right) C_{z} \sin \left(k_{y} y\right) F_{z} \cos \left(k_{z} z\right)
\end{gathered}
$$

Renomeando as constantes,

$$
\begin{aligned}
& v_{x}=N_{x} \cos \left(k_{x} x\right) \sin \left(k_{y} y\right) \sin \left(k_{z} z\right) \\
& v_{y}=N_{y} \sin \left(k_{x} x\right) \cos \left(k_{y} y\right) \sin \left(k_{z} z\right) \\
& v_{z}=N_{z} \sin \left(k_{x} x\right) \sin \left(k_{y} y\right) \cos \left(k_{z} z\right)
\end{aligned}
$$

$$
\text { Com } x=L_{x} \text {, }
$$

$$
0=N_{x} \cos \left(k_{x} L_{x}\right) \sin \left(k_{y} y\right) \sin \left(k_{z} z\right)
$$


Portanto, é necessário ter $\mathrm{k}_{\mathrm{x}} \mathrm{L}_{\mathrm{x}}=\mathrm{n} \pi$. Ou seja,

$$
k_{x}=\frac{l \pi}{L_{x}} \quad \text { onde } l=0,1,2,3, \ldots
$$

Analogamente,

$$
\begin{array}{ll}
k_{y}=\frac{m \pi}{L_{y}} & \text { onde } m=0,1,2,3, \ldots \\
k_{z}=\frac{n \pi}{L_{z}} & \text { onde } n=0,1,2,3, \ldots
\end{array}
$$

Sendo assim, da Equação (14) se obtém

$$
k=\sqrt{\frac{l^{2} \pi^{2}}{L_{x}^{2}}+\frac{m^{2} \pi^{2}}{L_{y}^{2}}+\frac{n^{2} \pi^{2}}{L_{z}^{2}}}
$$

Portanto,

$$
\omega_{l, m, n}=c k_{l, m, n}=\pi c \sqrt{\left(\frac{l}{L_{x}}\right)^{2}+\left(\frac{m}{L_{y}}\right)^{2}+\left(\frac{n}{L_{z}}\right)^{2}}
$$

A energia de ponto zero entre as placas, segundo a Equação (2), é dada por

(2) $\frac{1}{2} \sum_{l, m, n} \hbar \omega_{l, m, n}=\pi \hbar c \sum_{l, m, n} \sqrt{\left(\frac{l}{L_{x}}\right)^{2}+\left(\frac{m}{L_{y}}\right)^{2}+\left(\frac{n}{L_{z}}\right)^{2}}$

O fator 2 vem do fato de existirem duas polarizações possiveis para o campo eletromagnético quando $l, m, n \neq 0$. Quando um dos coeficientes for nulo, a contribuição do respectivo coeficiente nulo deve ser dividida por dois [3]. Percebe-se isto derivando a Equação (26) obtendo

$$
k_{x} v_{x}+k_{y} v_{y}+k_{z} v_{z}=0
$$

A Equação acima mostra que um dos $k$ 's é combinação dos outros dois. Sendo assim, existem apenas duas polarizações para todos os $k$ 's diferentes de zero e apenas uma polarização se algum deles for zero.

A situação física de interesse corresponde a Figura 2 e pode ser obtida a partir da Figura 1 no limite em que se faz $L_{x}=L_{y}=L$ e $L_{z}=d$ com $L \gg d$. Portanto, a energia de ponto zero entre as placas será dada por

$$
\text { (2) } \frac{1}{2} \sum_{l, m, n} \hbar \omega_{l, m, n}=\pi \hbar c \sum_{l, m, n} \sqrt{\left(\frac{l}{L}\right)^{2}+\left(\frac{m}{L}\right)^{2}+\left(\frac{n}{d}\right)^{2}}
$$

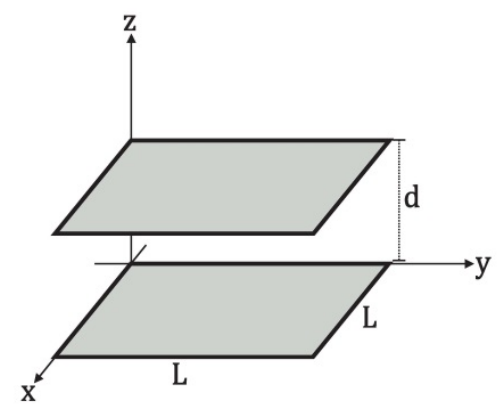

Figura 2 - Placas condutoras paralelas de área $L^{2}$ separadas por uma distância $d$. Assume-se que a placa inferior está no plano $x y$ na origem do sistema.

Para $L$ muito grande pode-se considerar $k_{x}$ e $k_{y}$ como variáveis contínuas [2]. Sendo assim, o somatório sobre $l$ e $m$ pode ser substituído por uma integral:

$$
\sum_{l, m} \rightarrow\left(\frac{L}{\pi}\right)^{2} \iint d k_{x} d k_{y}
$$

Portanto,

$$
\begin{aligned}
& E(d)=(2) \frac{1}{2} \sum_{l, m, n} \hbar \omega_{l, m, n} \\
& \rightarrow \frac{L^{2}}{\pi^{2}}(\hbar c) \sum_{n} \iint_{0}^{\infty}\left[\sqrt{k_{x}^{2}+k_{y}^{2}+\left(\frac{n \pi}{d}\right)^{2}}\right] d k_{x} d k_{y}
\end{aligned}
$$

Da mesma forma, para $L_{z}=d$ muito grande, a soma em $n$ pode se substituída por uma integral [2]:

$$
\sum_{n} \rightarrow \frac{d}{\pi} \int d k_{z}
$$

Sendo assim,

$$
E(\infty)=\frac{L^{2}}{\pi^{2}}(\hbar c) \frac{d}{\pi} \iiint_{0}^{\infty}\left[\left[\sqrt{k_{x}^{2}+k_{y}^{2}+k_{z}^{2}}\right]\right] d k_{x} d k_{y} d k_{z}
$$

A energia potencial do sistema quando as placas são separadas por uma distância $d$ é $U(d)=E(d)-E(\infty)$. Está é a energia de interação entre duas placas paralelas separadas por uma distância $d$. Logo,

$$
U(d)=\frac{L^{2}}{\pi^{2}}(\hbar c)\left\{\sum_{n} \iint_{0}^{\infty}\left[\sqrt{k_{x}^{2}+k_{y}^{2}+\left(\frac{n \pi}{d}\right)^{2}}\right] d k_{x} d k_{y}-\frac{d}{\pi} \iiint_{0}^{\infty}\left[\left[\sqrt{k_{x}^{2}+k_{y}^{2}+k_{z}^{2}}\right]\right] d k_{x} d k_{y} d k_{z}\right\}
$$




\section{Encontro Científico de Física Aplicada}

Utilizando coordenadas polares no plano $k_{x} k_{y}$, onde $r$ é o raio polar e $\theta$ é o ângulo polar, com $d k_{x} d k_{y}=r d r d \theta$, a Equação (46) pode ser reescrita como

$$
\begin{array}{r}
U(d)=\frac{L^{2} \hbar c}{\pi^{2}}\left\{\sum_{n} \int_{0}^{\frac{\pi}{2}} \int_{0}^{\infty}\left[\sqrt{r^{2}+\left(\frac{n \pi}{d}\right)^{2}}\right] r d r d \theta-\frac{d}{\pi} \int_{0}^{\frac{\pi}{2}} \int_{0}^{\infty} \int_{0}^{\infty}\left[\left[\sqrt{r^{2}+k_{z}^{2}}\right]\right] r d r d \theta d k_{z}\right\} \\
=\frac{L^{2} \hbar c}{\pi^{2}} \frac{\pi}{2}\left\{\sum _ { n } \int _ { 0 } ^ { \infty } \left[\sqrt{\left.\left.r^{2}+\frac{n^{2} \pi^{2}}{d^{2}}\right] r d r-\frac{d}{\pi} \int_{0}^{\infty} \int_{0}^{\infty}\left[\left[\sqrt{r^{2}+k_{z}^{2}}\right]\right] r d r d k_{z}\right\}}\right.\right.
\end{array}
$$

Para limitar o comportamento da integral, se introduz uma função $f(k)=f\left(\sqrt{r^{2}+k_{z}^{2}}\right)$. Fisicamente, as placas exercem pouca influência por não oferecerem obstáculo para ondas com comprimento de onda pequeno (grandes valores de $k$ ), da ordem de $1 / a_{0}$, onde $a_{0}$ é o raio de Bohr. Este efeito é conhecido como divergência ultravioleta [5]. Com o intuito de contornar essa divergência se faz

$$
f(k)= \begin{cases}1 & \text { para } k<k_{m} \\ 0 & \text { para } k \gg k_{m}\end{cases}
$$

Portanto,

$$
\begin{aligned}
& U(d)=\frac{L^{2} \hbar c}{2 \pi}\left\{\sum_{n} \int_{0}^{\infty}\left[\sqrt{r^{2}+\frac{n^{2} \pi^{2}}{d^{2}}}\right] f\left(\sqrt{r^{2}+\frac{n^{2} \pi^{2}}{d^{2}}}\right) r d r-\frac{d}{\pi} \int_{0}^{\infty} \int_{0}^{\infty}\left[\left[\sqrt{r^{2}+k_{z}^{2}}\right]\right] f\left(\sqrt{r^{2}+\frac{n^{2} \pi^{2}}{d^{2}}}\right) r d r d k_{z}\right\} \\
& =\frac{L^{2} \hbar c}{2 \pi}\left\{\sum_{n} \int_{0}^{\infty}\left[\frac{\pi}{d} \sqrt{\frac{d^{2} r^{2}}{\pi^{2}}+n^{2}}\right] f\left(\frac{\pi}{d} \sqrt{\frac{d^{2} r^{2}}{\pi^{2}}+n^{2}}\right) r d r-\frac{d}{\pi} \int_{0}^{\infty} \int_{0}^{\infty}\left[\frac{\pi}{d}\left[\sqrt{\frac{d^{2} r^{2}}{\pi^{2}}+\frac{d^{2} k_{z}^{2}}{\pi^{2}}}\right]\right] f\left(\frac{\pi}{d}\left[\sqrt{\frac{d^{2} r^{2}}{\pi^{2}}+\frac{d^{2} k_{z}^{2}}{\pi^{2}}}\right]\right) r d r d k_{z}\right\}
\end{aligned}
$$

Fazendo as substituições

$$
x=\frac{d^{2} r^{2}}{\pi^{2}} \rightarrow d x=\frac{2 d^{2}}{\pi^{2}} r d r \quad \text { e } \quad \kappa=\frac{d k_{z}}{\pi} \rightarrow d \kappa=\frac{d}{\pi} d k_{z}
$$

a Equação (49) se torna

$$
U(d)=\frac{L^{2} \hbar c}{2 \pi}\left\{\sum_{n} \int_{0}^{\infty}\left[\frac{\pi}{d} \sqrt{x+n^{2}}\right] f\left(\frac{\pi}{d} \sqrt{x+n^{2}}\right) \frac{\pi^{2}}{2 d^{2}} d x-\frac{d}{\pi} \int_{0}^{\infty} \int_{0}^{\infty}\left[\frac{\pi}{d}\left[\sqrt{x+\kappa^{2}}\right]\right] f\left(\frac{\pi}{d}\left[\sqrt{x+\kappa^{2}}\right]\right) \frac{\pi^{2}}{2 d^{2}} d x \frac{\pi}{d} d \kappa\right\}
$$

Simplificando,

$$
U(d)=\frac{L^{2} \hbar c}{2 \pi} \frac{\pi^{3}}{d^{3}}\left\{\sum_{n} \int_{0}^{\infty}\left[\sqrt{x+n^{2}}\right] f\left(\frac{\pi}{d} \sqrt{x+n^{2}}\right) d x-\int_{0}^{\infty} \int_{0}^{\infty}\left[\left[\sqrt{x+\kappa^{2}}\right]\right] f\left(\frac{\pi}{d}\left[\sqrt{x+\kappa^{2}}\right]\right) d x d \kappa\right\}
$$

Fazendo

$$
F(\kappa)=\int_{0}^{\infty}\left[\left[\sqrt{x+\kappa^{2}}\right]\right] f\left(\frac{\pi}{d}\left[\sqrt{x+\kappa^{2}}\right]\right) d x
$$

a Equação (51) fica

$$
U(d)=\frac{\pi^{2} \hbar c}{4 d^{3}} L^{2}\left\{\frac{1}{2} F(0)+\sum_{n=1}^{\infty} F(n)-\int_{0}^{\infty} F(\kappa) d \kappa\right\}
$$

Lembrando que o fator meio multiplicando $F(0)$ na Equação (53) refere-se a uma única liberdade de polarização quando $n=0$. De acordo com a fórmula do somatório de Euler-Maclaurin ${ }^{1}$,

$$
\begin{aligned}
\sum_{n=1}^{\infty} F(n)-\int_{0}^{\infty} F(\kappa) & d \kappa \\
& =\frac{1}{2} F(0)-\frac{1}{12} F^{\prime}(0)+\frac{1}{720} F^{\prime \prime \prime}(0) \cdots
\end{aligned}
$$

Derivando a Equação (52), porém fazendo a substituição $u=x+\kappa^{2}$, se obtém 


$$
F(\kappa)=\int_{\kappa^{2}}^{\infty} \sqrt{u} f\left(\frac{\pi}{d} \sqrt{u}\right) d u
$$

Integrando,

$$
\begin{aligned}
F(\kappa) & =\left.\left[\frac{2}{3} u^{3 / 2} f\left(\frac{\pi}{d} u^{1 / 2}\right)\right]\right|_{\kappa^{2}} ^{\infty} \\
& =-\frac{2}{3} \kappa^{3} f\left(\frac{\pi}{d} \kappa\right)
\end{aligned}
$$

Portanto,

$$
\begin{array}{ll}
F^{\prime}(\kappa)=-2 \kappa^{2} f\left(\frac{\pi}{d} \kappa\right) & \rightarrow F^{\prime}(0)=0 \\
F^{\prime \prime}(\kappa)=-4 \kappa f\left(\frac{\pi}{d} \kappa\right)-2 \kappa^{2} f^{\prime}\left(\frac{\pi}{d} \kappa\right) & \rightarrow F^{\prime \prime}(0)=0 \\
F^{\prime \prime \prime}(\kappa)=-4 f\left(\frac{\pi}{d} \kappa\right)-4 \kappa f^{\prime}\left(\frac{\pi}{d} \kappa\right) & \rightarrow F^{\prime \prime \prime}(0)=-4
\end{array}
$$

As demais derivadas de ordens superiores serão nulas assumindo que todas as derivadas da função de corte desaparecem quando $\kappa=0$ [2]. Portanto, a Equação (54) fica

$$
\sum_{n=1}^{\infty} F(n)-\int_{0}^{\infty} F(\kappa) d \kappa=\frac{1}{2} F(0)-\frac{4}{720}
$$

Substituindo a Equação (57) na Equação (53), segue que

$$
\begin{gathered}
U(d)=\frac{\pi^{2} \hbar c}{4 d^{3}} L^{2}\left\{\frac{1}{2} F(0)+\frac{1}{2} F(0)-\frac{4}{720}\right\} \\
U(d)=-\frac{\pi^{2} \hbar c}{720 d^{3}} L^{2}
\end{gathered}
$$

Como $F(d)=-\vec{\nabla} U(d)$, então

$$
F(d)=-\frac{\pi^{2} \hbar c}{240 d^{4}} L^{2}
$$

Portanto, a força atrativa por unidade de área entre as placas é

$$
F(d)=-\frac{\pi^{2} \hbar c}{240 d^{4}}
$$

Sendo assim, existe uma força atrativa entre duas placas metálicas que independe do material das placas que pode ser interpretada como uma pressão de ponto zero das ondas eletromagnéticas [3] que pode ser interpretada como flutuações da energia de ponto zero das ondas eletromagnéticas.

\section{Agradecimentos}

Os autores agradecem a CAPES pelo apoio financeiro.

\section{Referências}

[1] CASIMIR, H. B. G.; POLDER, D. The Influence of Retardation on the London-van der Waals Forces. Physical Review, v. 73, n. 4, p. 360-372, fev. 1948. ISSN 0031-899X.

[2] MILONNI, P. W. The Quantum Vacuum. 1. ed. San Diego: Academin Press, Inc, 1994. 522 p.

[3] CASIMIR, H. B. G. On the attraction between two perfectly conducting plates. Proc. K. Ned. Akad. Wet., v. 51, n. April, p. 793-795, 1948.

[4] ABRAMOWITZ, M.; STEGUN, I. A. Handbook of Mathematical Functions. 9. ed. New York: Dover Publications, Inc., 1970. 1072 p.

[5] DILEM, B. B. Os efeitos das condições de contorno na eletrodinâmica escalar e o efeito Casimir para $\mathrm{N}$ regiões de largura finita $\mathrm{e}$ diferentes potenciais, Vitória, Tese (Doutorado em Física), 2012. 